\title{
Yearly variations in the low-latitude topside ionosphere
}

\author{
G. J. Bailey ${ }^{1}$, Y. Z. Su ${ }^{1}$, K.-I. Oyama ${ }^{2}$ \\ ${ }^{1}$ Department of Applied Mathematics, The University of Sheffield, Sheffield S3 7RH, UK \\ E-mail: g.bailey@sheffield.ac.uk \\ ${ }^{2}$ Institute of Space and Astronautical Science, 3-1-1 Yoshinodai, Sagamihara, Kanagawa 229, Japan \\ Received: 31 May 1999 / Revised: 22 September 1999 / Accepted: 24 September 1999
}

\begin{abstract}
Observations made by the Hinotori satellite have been analysed to determine the yearly variations of the electron density and electron temperature in the lowlatitude topside ionosphere. The observations reveal the existence of an equinoctial asymmetry in the topside electron density at low latitudes, i.e. the density is higher at one equinox than at the other. The asymmetry is hemisphere-dependent with the higher electron density occurring at the March equinox in the Northern Hemisphere and at the September equinox in the Southern Hemisphere. The asymmetry becomes stronger with increasing latitude in both hemispheres. The behaviour of the asymmetry has no significant longitudinal and magnetic activity variations. A mechanism for the equinoctial asymmetry has been investigated using CTIP (coupled thermosphere ionosphere plasmasphere model). The model results reproduce the observed equinoctial asymmetry and suggest that the asymmetry is caused by the north-south imbalance of the thermosphere and ionosphere at the equinoxes due to the slow response of the thermosphere arising from the effects of the global thermospheric circulation. The observations also show that the relationship between the electron density and electron temperature is different for daytime and nighttime. During daytime the yearly variation of the electron temperature has negative correlation with the electron density, except at magnetic latitudes lower than $10^{\circ}$. At night, the correlation is positive.
\end{abstract}

Key words: Ionosphere (equatorial ionosphere; ionosphere-atmosphere interactions; plasma temperature and density)

\section{Introduction}

Yearly variations of the Earth's ionosphere can be represented by a superposition of annual, seasonal and semiannual components, and the yearly average. Both the annual and seasonal components have a 12-month cycle with maxima and minima around the June and December solstices; the annual component has the same phase in both hemispheres whilst the seasonal components, in each hemisphere, are 6 months out of phase. The semiannual component has a 6-month cycle with maxima around the March and September equinoxes.

Recently, Su et al. (1998) have compared the electron densities in the topside ionosphere observed by the Hinotori satellite at the June and December solstices. They found that the behaviour of the annual and seasonal variations in the topside ionosphere is rather different to that reported previously for the ionospheric F region (e.g. Torr and Torr, 1973). In the present study, a 12-month period of electron density and electron temperature data observed by the Hinotori satellite is analysed to investigate the yearly variations of the topside ionosphere. As we have already carried out an investigation of the behaviour at the June and December solstices ( $\mathrm{Su}$ et al., 1998), in the present study we concentrate on the behaviour at the equinoxes.

Observations covering a wide variety of physical parameters show the existence of equinoctial asymmetries in the ionosphere and thermosphere, i.e. the parameters are larger at one equinox than at the other. Essex (1977) and Titheridge and Buonsanto (1983) found an equinoctial asymmetry in the observations of the ionospheric total electron content (TEC) and that the behaviour of the asymmetry depends on location and solar activity. Recently, Feichter and Leitinger (1997) have reported that the equinoctial asymmetries in TEC and F2-layer peak electron density (NmF2) change with the 11-year solar cycle. The altitude dependencies of the asymmetry in the ionosphere at low latitudes have been investigated by Balan et al. (1998) using data from 
the Japanese MU radar. Balan et al. (1998) found that the strength of equinoctial asymmetry in electron density increases with increasing altitude. The existence of an equinoctial asymmetry in the nighttime thermospheric neutral wind at high latitudes has been reported by Aruliah et al. (1996a). In Aruliah et al. (1996b, 1997), it is suggested the asymmetry arises from the diurnal variation in the cross polar cap potential difference due to the annual and diurnal variation in the orientation of the magnetosphere with respect to the interplanetary magnetic field.

Observations presented show that there are significant equinoctial asymmetries in the electron density and electron temperature of the low-latitude topside ionosphere. The latitudinal, longitudinal and magnetic activity variations of the asymmetries are also investigated. A source mechanism for the asymmetries is investigated using CTIP (coupled thermosphere ionosphere plasmasphere model, Millward et al., 1996a).

\section{Data analysis and results}

The Hinotori satellite was launched into a near-circular orbit at $600 \mathrm{~km}$ altitude with an inclination of $31^{\circ}$ in February 1981 and it operated until June 1982. The orbital period of the satellite was about 90 min. Since the memory of the recorder on board the satellite was limited, only 4 to 6 orbits of data per day were transmitted to the data acquisition station at the Kagoshima Space Center. The electron density and electron temperature were measured by an impedance probe in an upper resonance mode (Watanabe and Oya, 1986) and a resonance rectification probe (Hirao and Oyama, 1970), respectively. The relative error of the electron density measurements is about $10 \%$ and the maximum error of the electron temperature is about $50 \mathrm{~K}$. It should be pointed out that normally the F2 layer peak of the ionosphere is lower than the altitude of the satellite $(600 \mathrm{~km})$, although the peak might occasionally move to above $600 \mathrm{~km}$ around equator during the postsunset period (Su et al., 1997).

To obtain monthly averaged values of electron density and temperature covering each month of a year, we have used the data obtained over the period February 1981 to February 1982. The data from the months of February 1981 and February 1982 were combined to provide the monthly averaged values for February because only a few days of data are available for February 1981 (data collection did not commence until 23 February, 1981). Although the time sequence of the data is from February 1981 to February 1982, we present our results in the order of January to December. The number of days, the number of orbits on which data are available, and the monthly averaged F10.7 for these days are listed in Table 1. It can be seen from Table 1 that the values of the averaged F10.7 are lower around the solstices than around the equinoxes. These differences in F10.7 might have some effect on the yearly variations of the ionosphere. However, the differences of the monthly averaged values between the March and
Table 1. Number of days and orbits in which the data are available and the monthly-averaged F10.7 for each month

\begin{tabular}{llll}
\hline Month & $\begin{array}{l}\text { Number of } \\
\text { days }\end{array}$ & $\begin{array}{l}\text { Number of } \\
\text { orbits }\end{array}$ & F10.7 \\
\hline January & 21 & 116 & 177 \\
February & 32 & 167 & 214 \\
March & 23 & 109 & 205 \\
April & 24 & 95 & 230 \\
May & 28 & 137 & 198 \\
June & 27 & 133 & 161 \\
July & 25 & 113 & 200 \\
August & 21 & 105 & 217 \\
September & 25 & 122 & 223 \\
October & 27 & 135 & 222 \\
November & 24 & 124 & 206 \\
December & 23 & 118 & 206 \\
\hline
\end{tabular}

September equinoxes should not be significantly affected by the variations in solar activity since the difference in F10.7 between the two equinoxes is small.

For each month, the electron density and temperature are averaged into bins of $5^{\circ}$ magnetic latitude for daytime hours (09:00-17:00 LT) and for nighttime hours (23:00-04:00 LT). The yearly variations of the electron density and electron temperature for each latitude bin are displayed in Figs. 1 (daytime) and 2 (nighttime). The data observed at all longitudes and magnetic activity levels are included in the results displayed in these figures. The central latitude of each latitude bin is given in each panel of each figure. For clarity, the error bars are only shown for the equatorial and Southern-Hemisphere curves. The errors bars for the Northern Hemisphere are of similar magnitude to those of the Southern Hemisphere.

Figure 1a shows that, in general, the yearly variation of the daytime electron density has maxima around the equinoxes and minima around the solstices. This feature is the semiannual anomaly, which has been observed in the ionospheric F region (e.g. Torr and Torr, 1973). However, as Table 1 shows, F10.7 is higher at both the equinoxes than at either of the solstices and these differences in F10.7 might make a contribution to the differences in the electron density at the equinoxes and solstices. Figure 1a also shows a strong annual anomaly, i.e. the electron density being higher at the December solstice than at the June solstice. As a result of a superposition of the annual anomaly and seasonal variation, the electron density is much higher at the December solstice than at the June solstice in the Southern Hemisphere and is similar at both solstices in the Northern Hemisphere. The behaviour and a possible source mechanism for the annual and seasonal components have been investigated in some detail by $\mathrm{Su}$ et al. (1998).

Fig. 1a. Yearly variations of the daytime (09:00-17:00 LT) averaged electron density at the magnetic equator and at magnetic latitudes $5^{\circ}$, $10^{\circ}, 15^{\circ}, 20^{\circ}$ and $25^{\circ}$; Southern Hemisphere (solid curves) and Northern Hemisphere (dashed curves). b Same as a except for the electron temperature 
DAYTIME

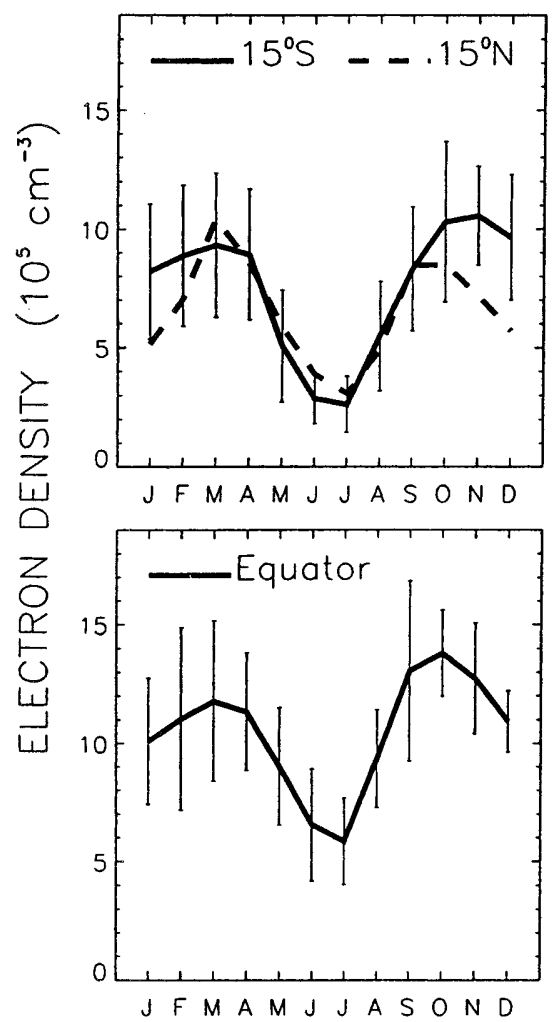

a

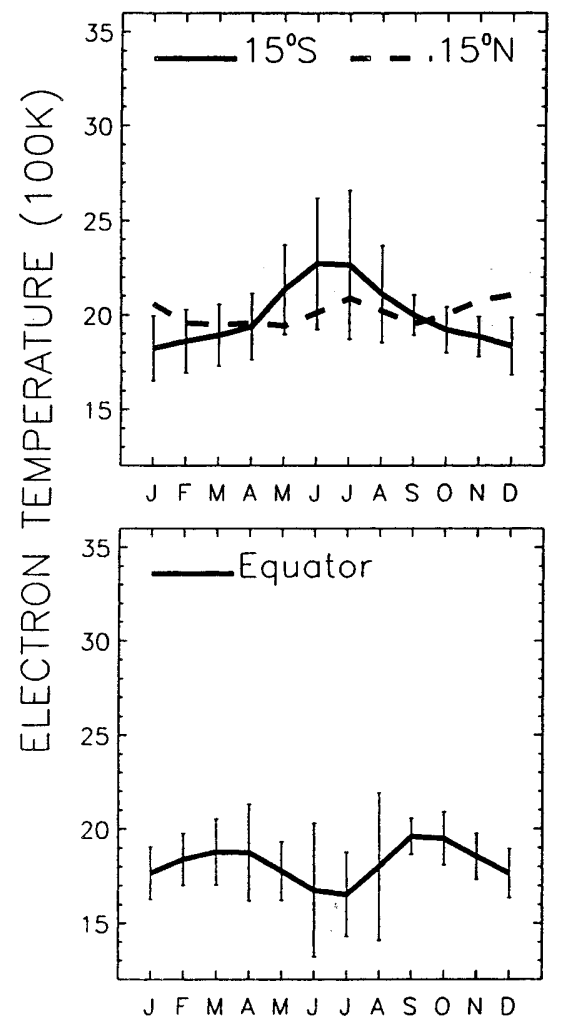

b
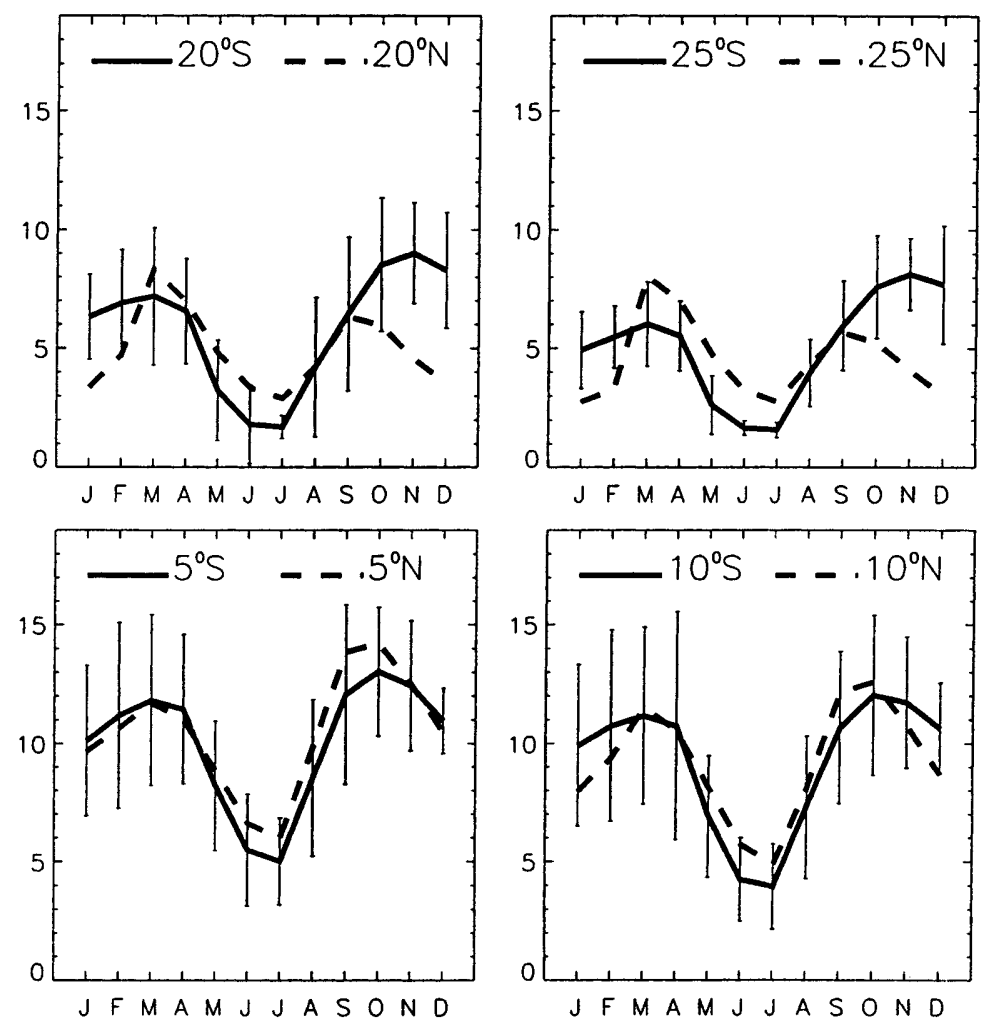

MONTH

DAYTIME
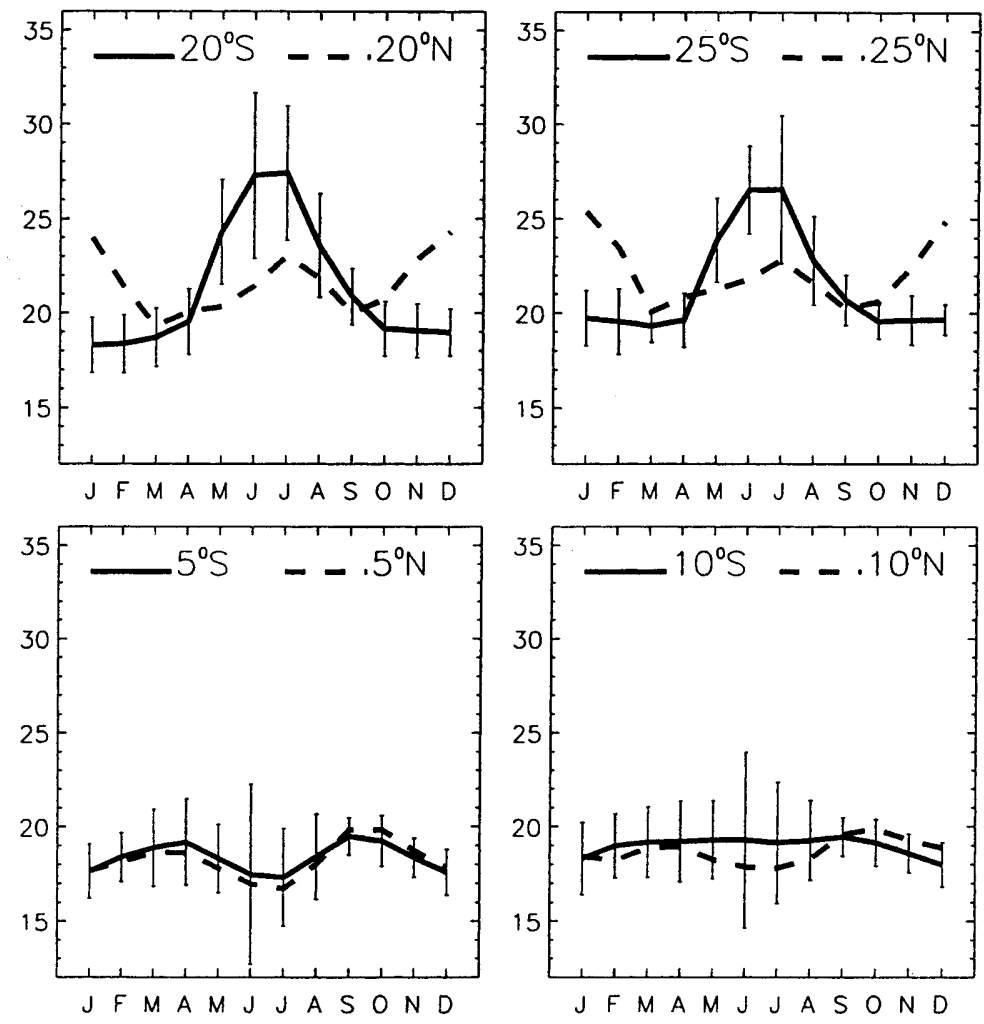

MONTH 
A notable feature seen in Fig. 1a is the existence of an equinoctial asymmetry in the electron density, i.e. the electron density is higher at one equinox than at the other. When the latitude is greater than $10^{\circ}$, in the Southern Hemisphere, the electron density is higher at the September equinox than at the March equinox while in the Northern Hemisphere the higher density occurs at the March equinox. At greater latitudes, the asymmetry becomes stronger with increasing latitude. At latitude $25^{\circ}$, in both hemispheres, the electron density at one equinox is about $40 \%$ higher than at the other. The electron density is similar at both equinoxes around latitudes of $\pm 10^{\circ}$ but the density is higher at the September equinox than at the March equinox around magnetic equator. The feature around the magnetic equator might be partially affected by some unknown bias in the data. Month-to-month variations of solar activity and magnetic activity are not thought to be the reason for the equinoctial difference in the electron densities at the latitudes above $10^{\circ}$ since the higher density occurs at different equinoxes in different hemispheres.

As shown in Fig. 1b, at the magnetic equator, the yearly variation in the daytime electron temperature has peaks around the equinoxes and minima around the solstices. However, with increasing latitude, the temperature increases more rapidly at the solstices than at the equinoxes. As a result, at the higher latitudes, the yearly variation in the electron temperature has a negative correlation with the electron density. In the Northern Hemisphere, the two peaks in electron temperature around the solstices correspond to the two minima in the yearly variations of the electron density. In the Southern Hemisphere, the annual variation is dominant; only one electron temperature peak appears around the June solstice and at the December solstice the temperature is lowest or similar to that at the equinoxes. The low temperature at the December solstice results from the electron density at the December solstice being much higher than at the June solstice and similar to that at the equinoxes due to the superposition of the annual and seasonal variations (see Fig. 1a). In both hemispheres, the electron temperature is lower in summer than in winter. There is no significant difference in the electron temperature between the two equinoxes.

A comparison of Figs. 2a and 1a shows that, while the general features that occur during nighttime are similar to those that occur during daytime, the equinoctial asymmetry is stronger at night. At night, the feature that the higher density occurs at different equinoxes in different hemispheres can be seen at magnetic latitudes as low as $5^{\circ}$, while the feature cannot be seen during daytime when the latitude is lower than $15^{\circ}$. In the Southern/Northern Hemisphere, with increasing latitude, the electron density peak around the September/March equinox becomes stronger and moves slightly towards the summer. At the same time, the peaks that occur around the March/September equinox disappear. At latitude $25^{\circ}$, in the Southern/Northern hemisphere, the peak electron density at the September/
Fig. 2a. Same as Fig. 1a except for nighttime (23:00-04:00 LT). b Same as a except for the electron temperature

March equinox is about $100 \%$ higher than at the March/September equinox.

The yearly variation in the nighttime electron temperature (see Fig. 2b) has, in general, positive correlation with the electron density with the electron temperature being higher around the equinoxes than around the solstices. This feature is contrary to that seen during daytime at higher latitudes. The nighttime temperature at higher latitudes shows an equinoctial asymmetry with the temperature being lower at the March/September equinox than at the September/ March equinox in the Southern/Northern Hemisphere. The asymmetry in the electron temperature is much weaker than that occurring in the electron density.

To investigate the longitudinal variation of the equinoctial asymmetry, we have separated the data into three longitude sectors. The choice of longitudes for each sector has been based on the configuration of the geomagnetic field. The data have been averaged into bins of $10^{\circ}$ in magnetic latitude instead of the previously adopted value of $5^{\circ}$ to give more reliable average values for each longitude sector. The yearly variations of the daytime (09:00-17:00) average electron density for the three longitude sectors are shown in Fig. 3. It can be seen from Fig. 3 that, although there are longitudinal differences, for each longitudinal sector, the general features of the equinoctial asymmetry are similar to those shown in Fig. 1a. The equinoctial asymmetries with higher electron density around the March/September equinox in the Northern/Southern Hemisphere occur in each of the three longitude sectors at the higher latitudes. It is noted that, for the longitude sector $240-320^{\circ} \mathrm{E}$ in the Northern Hemisphere, the asymmetry is smaller than that occurring in the other longitude sectors.

In Aruliah et al. (1996b, 1997), it has been suggested that, at high latitudes for the same hemisphere, the neutral wind should be higher at one equinox in one longitude sector and at the other equinox in the longitude sector that differs by $180^{\circ}$. However, there is no data at high latitudes to test this suggestion. The longitude variations shown in Fig. 3 suggest that, for low latitudes, the behaviour of the equinoctial asymmetry is not significantly different to that in the longitude sector that differs by $180^{\circ}$. Thus the mechanism that Aruliah et al. (1996b, 1997) suggested does not extend to low-latitudes.

Geomagnetic activity effects have been investigated by separating the data into low $(A p \leq 15)$ and high $(A p>15)$ magnetic activity levels. The yearly variations of the daytime (09:00-17:00) average electron density for both magnetic activity levels are displayed in Fig. 4. This figure shows that the equinoctial asymmetry seen in Fig. 1a occurs during both low and high magnetic activity levels. However, there are indications in Fig. 4 to suggest that the equinoctial asymmetry is stronger during periods of low magnetic activity than during periods of high magnetic activity. 


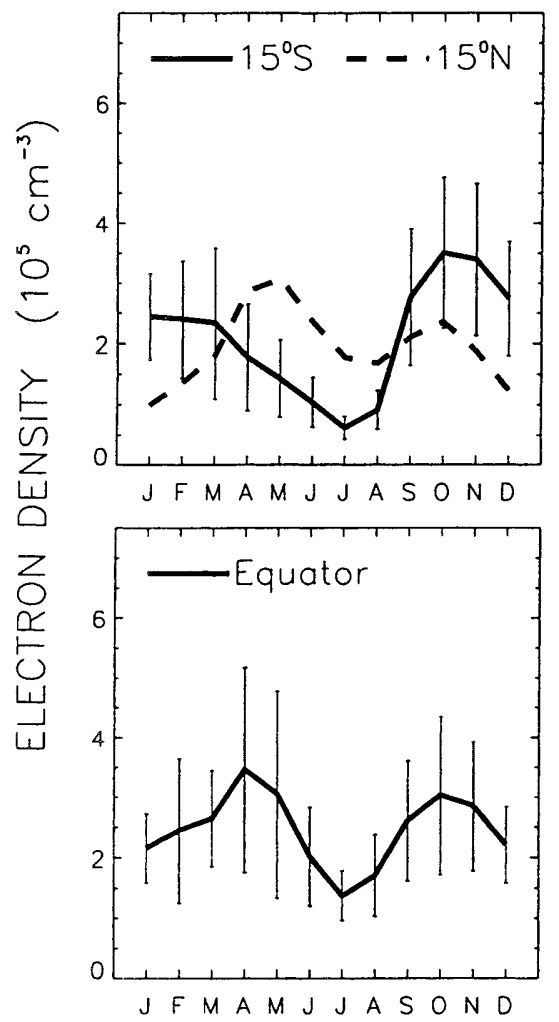

a

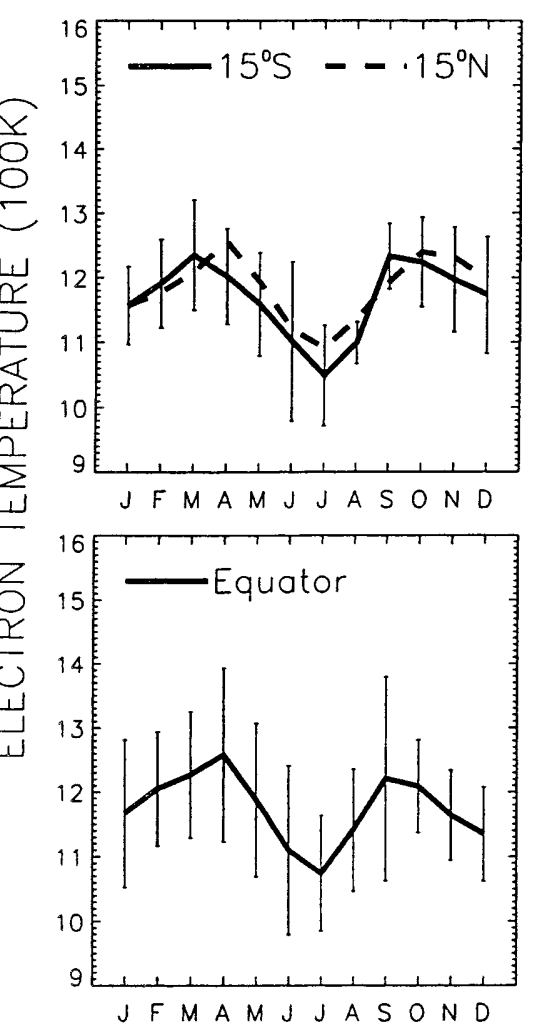

b
NIGHTTIME
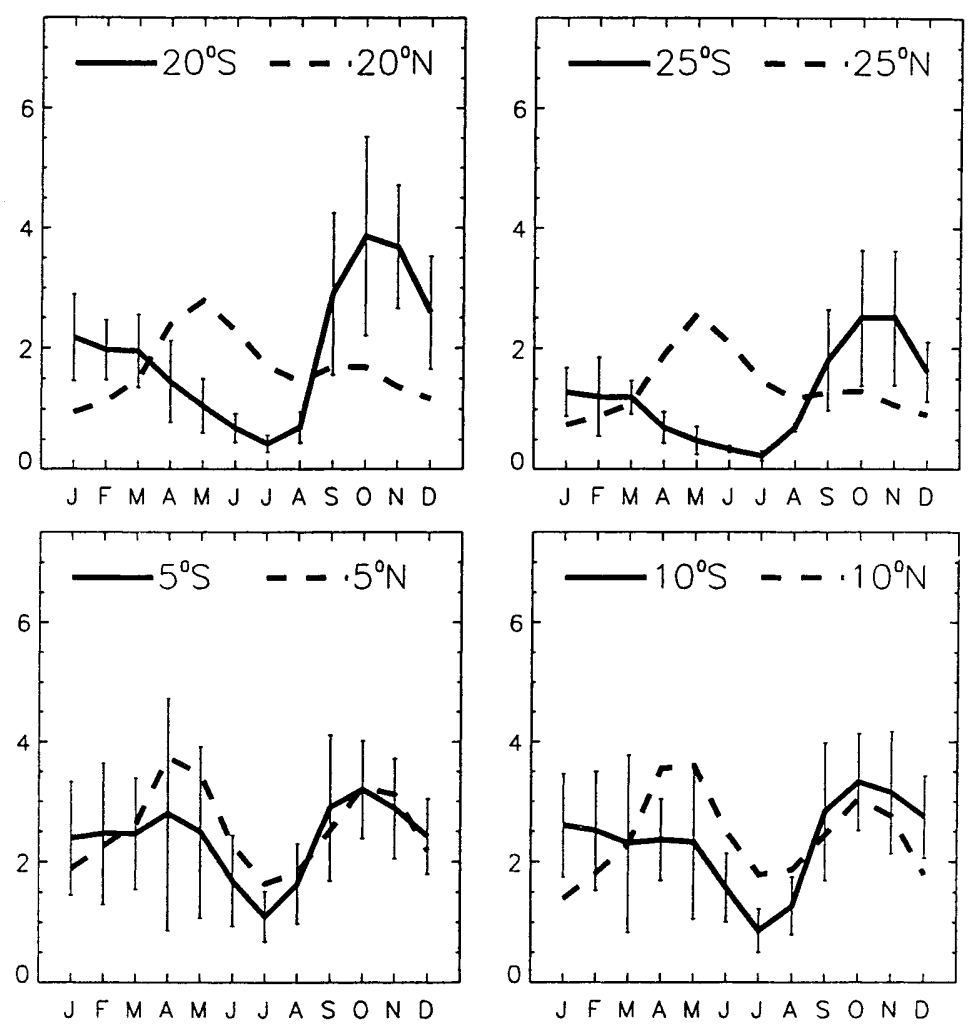

MONTH

NIGHTTIME
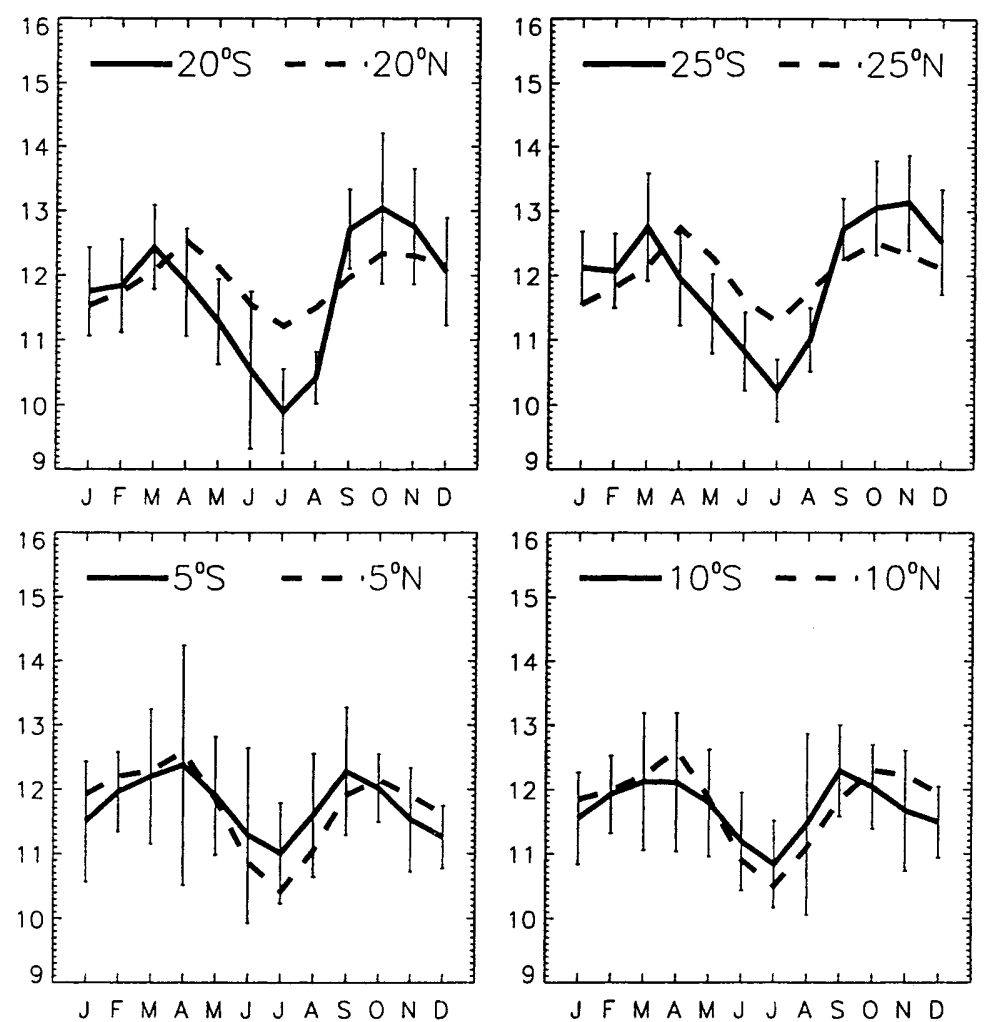

MONTH

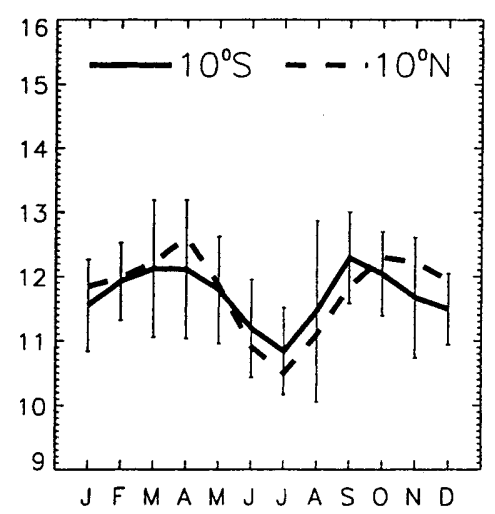



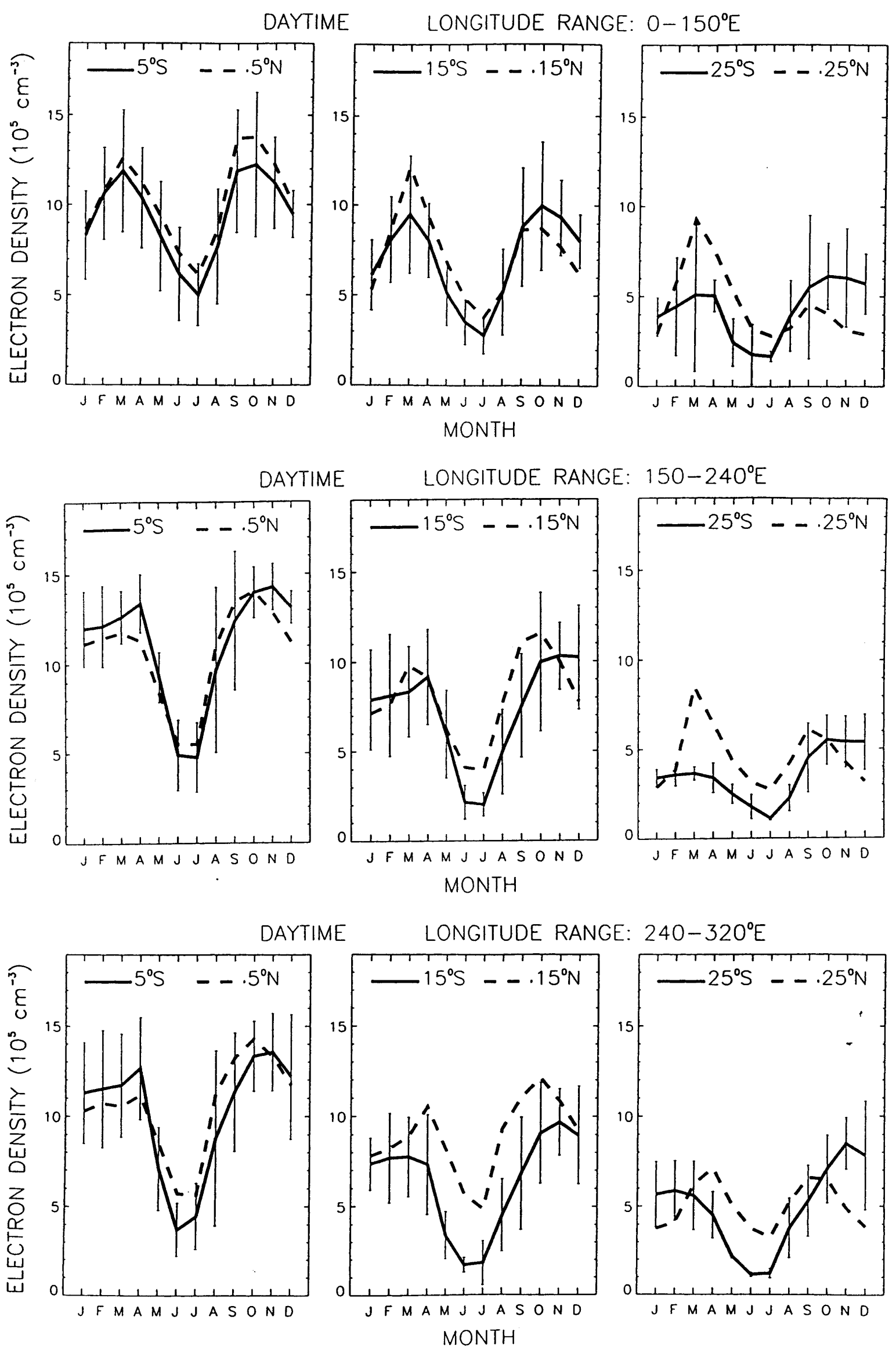
Fig. 3. Yearly variations of the daytime (09:00-17:00 LT) averaged electron density at magnetic latitudes $5^{\circ}, 15^{\circ}$ and $25^{\circ}$ for the longitudinal sectors $0-150^{\circ} \mathrm{E}$ (top panels), $150-240^{\circ} \mathrm{E}$ (middle panels) and $240-320^{\circ} \mathrm{E}$ (bottom panels); Southern Hemisphere (solid curves) and Northern Hemisphere (dashed curves)

\section{Model calculations and results}

The model used for the present study is CTIP, the coupled thermosphere ionosphere plasmasphere model (Millward et al., 1996a). This model has been developed during the past decade from the global thermosphere model of Fuller-Rowell and Rees (1980), the highlatitude ionosphere model of Quegan et al. (1982), and the mid and low-latitude ionosphere-plasmasphere model of Bailey et al. (1993). In CTIP, the thermosphere model and the ionosphere-plasmasphere model run concurrently and are fully coupled with respect to energy, momentum and continuity.

In previous studies, e.g. Millward et al. (1996b), it was realized that the model results could be affected by the initial conditions. To overcome this problem, Millward et al. (1996b) ran CTIP for twenty 24-h periods with the same day and model inputs in order to achieve a steady state condition. Running the model in this way is to assume that the position between the earth and the sun does not change over the 20-day period, which is not the case in reality. This method effectively loses the geophysical history. As a result, their model results produced no significant differences for the March and September equinoxes. In the present study, we approach this problem in a different way. To obtain the model results for the March and September equinoxes, we have simulated the passage of time. Thus we have run the model continuously for each day
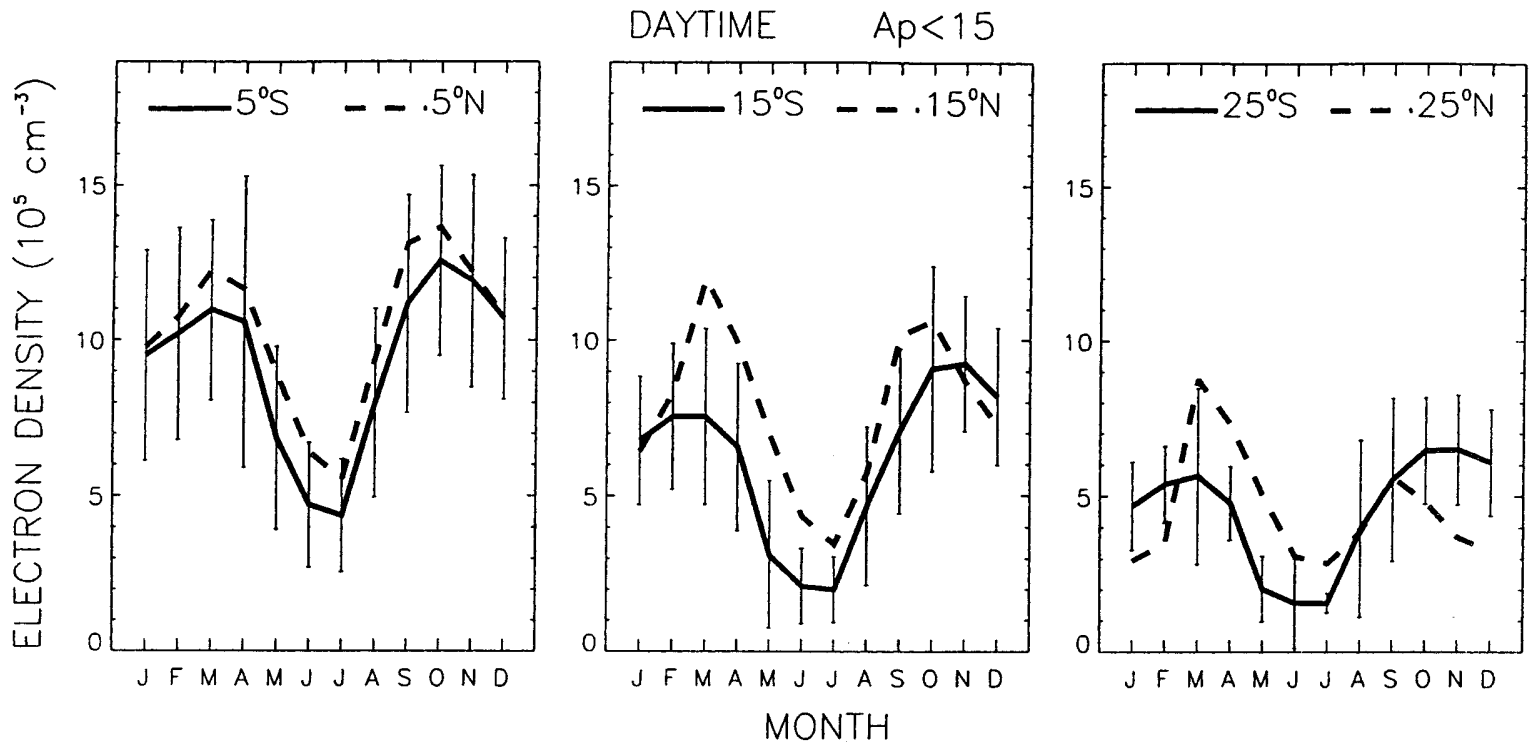

DAYTIME Ap $>15$
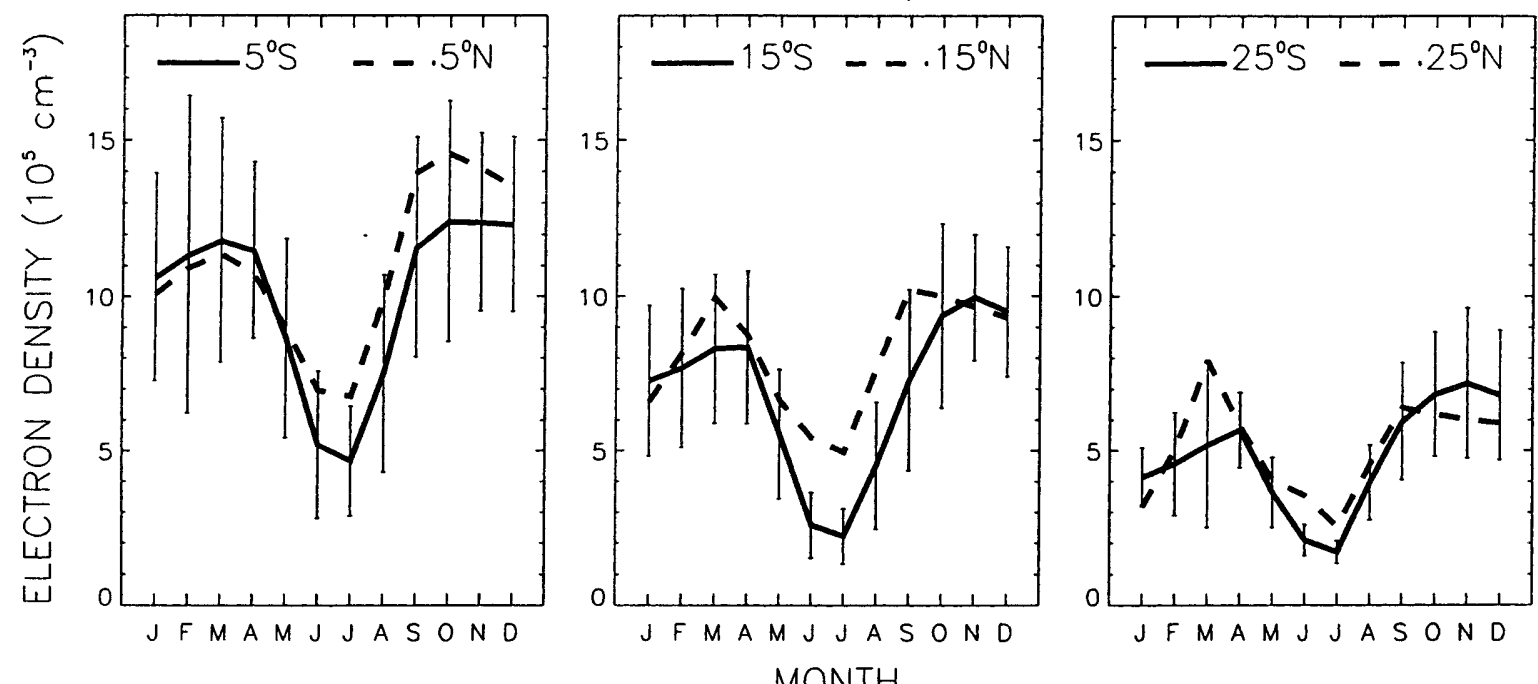

Fig. 4. Yearly variations of the daytime (09:00-17:00 LT) averaged electron density at magnetic latitudes $5^{\circ}, 15^{\circ}$ and $25^{\circ}$ Southern Hemisphere (solid curves) and Northern Hemisphere (dashed curves) for low (top panels) and high (bottom panels) magnetic conditions 
between the December solstice day and the March equinox day and between the June solstice day and the September equinox day, respectively. All the model calculations have been carried out for magnetically quiet $(A p=7)$ and high solar activity $(\mathrm{F} 10.7=180)$ conditions.

The latitude variations of the modelled electron density at $600 \mathrm{~km}$ altitude during daytime (14:00 LT) and nighttime (02:00 LT) at the March and September equinoxes are displayed in Fig. 5a and b, respectively. It can be seen that, during both daytime and nighttime in the Northern Hemisphere, the modelled electron densities are higher at the March equinox than at the September equinox. The equinox with the higher electron density changes near the magnetic equator, thus, in the Southern Hemisphere, the higher electron density occurs at the September equinox. These modelled
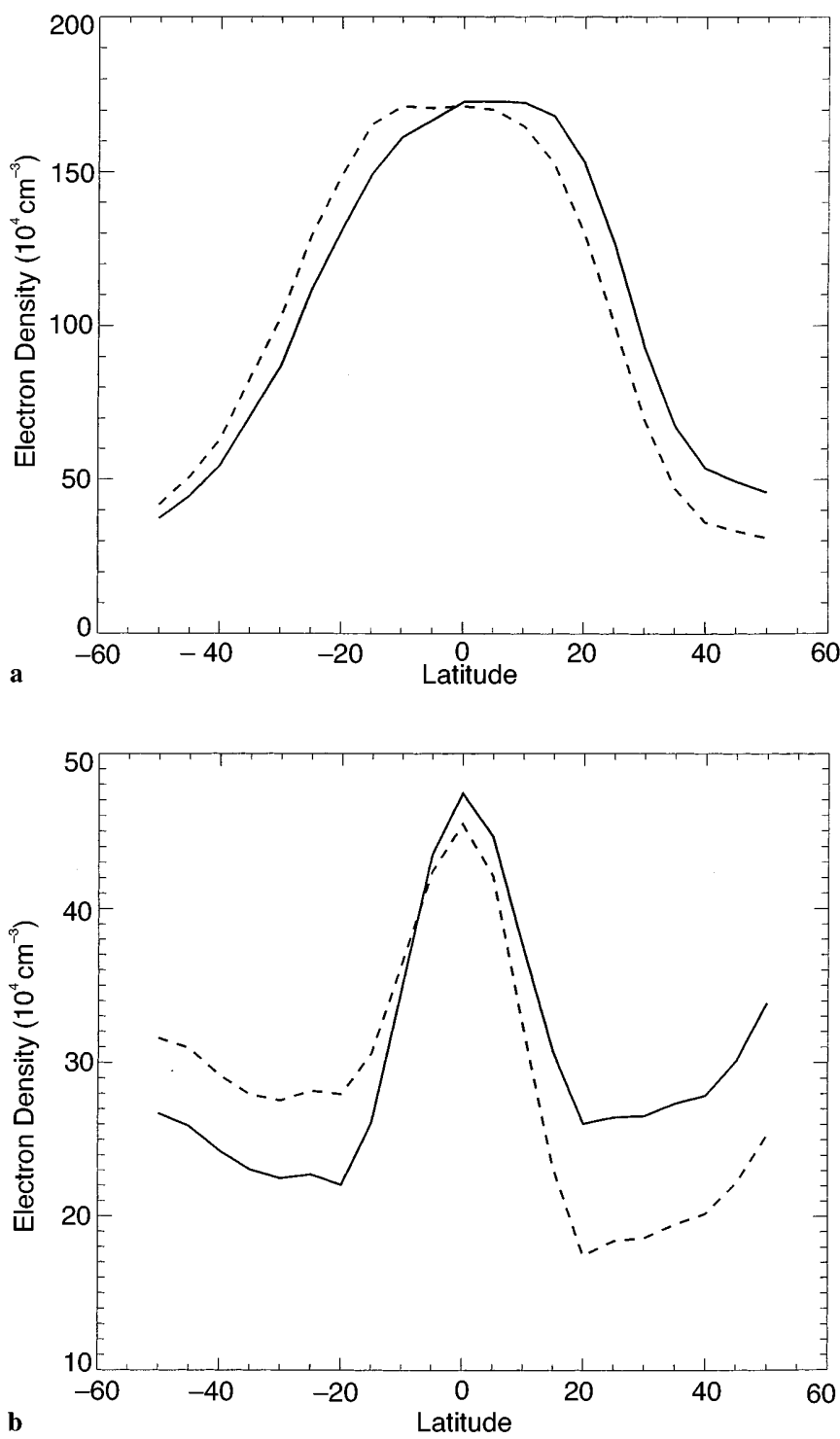

Fig. 5a. Latitude variations of the modelled electron density at March (solid curve) and September (dashed curve) during daytime. b Same as a except for nighttime equinoctial asymmetries and hemisphere dependencies are in good agreement with the observations. By comparing Fig. 5a, b, it can be seen that the percentage difference in the electron density between the two equinoxes is much larger during nighttime than during daytime. Again, this modelled feature is consistent with the observations that the equinoctial asymmetry is stronger during nighttime than during daytime.

The model results suggest that the equinoctial asymmetry at low latitudes is caused by the global thermosphere circulation. During the period when the season changes from solstice to equinox, the thermosphere is still winter-like in one hemisphere and summer-like in the other hemisphere. As thermospheric circulation changes the thermosphere slowly, there is still an imbalance between the hemispheres on equinoctial days even though the direct energy input to the thermosphere by the solar radiation is the same for both hemispheres. The thermospheric imbalance at the equinoxes gives rise to the imbalance in the ionosphere. Also, as summer and winter occur in opposite hemispheres at the December and June solstices, the ionospheric imbalance gives rise to higher and lower electron densities in opposite hemispheres at the March and September equinoxes.

\section{Discussion and summary}

Yearly variations of the topside ionosphere at low latitudes have been investigated using observations made by the Hinotori satellite. The observations show the existence of an equinoctial asymmetry in the lowlatitude topside ionosphere with the higher electron density changing from the Northern Hemisphere at the March equinox to the Southern Hemisphere at the September equinox. These features occur at all longitudes. While the behaviour of the asymmetry in the Northern Hemisphere shown in this study is in accord with the MU radar observations at Shigaraki (magnetic latitude $25^{\circ} \mathrm{N}$ ), Japan, and reported by Balan et al. (1998), the additional information obtained from the present study regarding the hemisphere dependency and the lack of significant longitudinal variation should assist in obtaining an understanding of a source mechanism for the asymmetry.

Our model calculations suggest that the equinoctial asymmetry is caused by the south-north imbalances of the thermosphere and ionosphere at the equinoxes due to the slow response of the thermosphere to the effects of thermospheric circulation. Observations showing the hemisphere dependency and the lack of significant longitudinal variation of the equinoctial asymmetry strongly support this suggested source mechanism for the asymmetry at low latitudes. Aruliah et al. (1996b, 1997) suggested that, at high latitudes, the equinoctial asymmetry in the nighttime neutral wind arises from the diurnal variation in the cross polar cap potential difference, which is caused by an annual and diurnal variation in the orientation of the magnetosphere with respect to the interplanetary magnetic field. The source mechanism of the asymmetry suggested by Aruliah et al. 
(1996b, 1997) implies that the amplitude of the asymmetry should decrease with decreasing latitude. Observational evidence given in this study strongly suggests that the proposed high-latitude source mechanism is not valid at low latitudes because the mechanism requires, for the same hemisphere, that the observed parameters should be higher at one equinox in one longitude sector and at the other equinox in the longitude sector that differs by $180^{\circ}$. This is contrary to the present lowlatitude observations. Feichter and Leitinger (1997) have reported that, at latitudes $45-65^{\circ}$ in the Northern Hemisphere the equinoctial asymmetry in TEC and NmF2 changes with the 11-year solar cycle. For the solar cycle when the ionospheric data from the Hinotori satellite were collected, Feichter and Leitinger (1997) showed that TEC and NmF2 are higher at the September equinox than at the March equinox. This is contrary to the observations made by the Hinotori satellite at low latitudes. Again, these differences seem to suggest that the mechanism for the equinoctial asymmetry is different for the low- and high-latitude regions.

The observations made by the Hinotori satellite also show that the semi-annual anomaly, i.e. the electron density being higher at the equinoxes than at the solstice, exists in the low-latitude topside ionosphere. The source of the semiannual anomaly is complicated. Recently, Fuller-Rowell (1998) used CTIM (coupled thermosphere ionosphere model), a version of CTIP, to investigate the semi-annual anomaly. Fuller-Rowell (1998) suggested that the semi-annual anomaly is caused by the "thermospheric spoon", i.e. the global thermospheric circulation at solstices raises the molecular nitrogen and oxygen densities and, therefore, reduces the atomic oxygen density compared with the equinoxes. Because the molecular gases and atomic gases control the loss rate and production rate of the plasma, respectively, a low atomic/molecular ratio gives rise to low electron densities in the ionosphere. The effect of the "thermospheric spoon" in the low-latitude ionosphere cannot be studied using CTIM because the model does not solve the equations of the low-latitude ionosphere in a self-consistent way. The effect of the "thermospheric spoon" on the low-latitude ionosphere needs to be investigated using CTIP.

Another interesting feature observed by the Hinotori satellite is that the relationship between the electron density and electron temperature is different during daytime and nighttime. While during daytime the yearly variation of the electron temperature has negative correlation with the electron density, except where the magnetic latitude is lower than $10^{\circ}$, at night, the temperature has positive correlation with the electron density. This behaviour of the electron density suggests that the electron density controls the electron temperature in different ways during daytime and nighttime. During daytime, at magnetic latitudes higher than $10^{\circ}$, high electron densities give rise to low electron temperatures, since for the same energy input from the sun, there are more electrons to be heated than when the electron density is low. Around the magnetic equator during daytime, the positive correlation of the electron temperature with the electron density suggests that, due to low heat capacity of the magnetic flux tube around the equator, the temperature of the ion gases is cooled to that of the neutral gas and that the electron temperature is not controlled by the density of the plasma but by the density of the neutral gas. At night, there are no energy sources to heat the plasma. When the plasma cools down, the higher electron density, which has a higher heating capacity, gives rise to a slow electron cooling and a positive correlation of the electron temperature with the electron density. It is likely that the reason for the asymmetry in the electron temperature at the equinoxes is negligible during daytime and only small at night is that the variability in the electron temperature is much smaller than that in the electron density $(\mathrm{Su}$ et al., 1995).

Acknowledgements. The authors would like to thank Professors H. Oya and T. Takahashi for their efforts in organising the electron density measurements on board the Hinotori satellite and Dr D. Idenden for his contribution to the scientific discussions. The work at Sheffield was supported by the Particle Physics and Astronomy Research Council (PPARC) under grants GR/L20238 and PPA/G/ O/1997/00691.

The Editor in chief thanks E. A. Essex and another referee for their help in evaluating this paper.

\section{References}

Aruliah, A. L., A. D. Farmer, D. Rees, and U. Brandstrom, The seasonal behaviour of high-latitude thermospheric winds and ion velocities observed over one solar cycle, J. Geophys. Res., 101, 15 701-15 711, 1996a.

Aruliah, A. L., A. D. Farmer, T. J. Fuller-Rowell, M. N. Wild, M. Hapgood, and D. Rees, An equinoctial asymmetry in the highlatitude thermosphere and ionosphere, J. Geophys. Res., 101, $15713-15722,1996 b$.

Aruliah, A. L., J. Schoenorf, A. D. Aylward, and M. N. Wild, Modelling the high-latitude equinoctial asymmetry, J. Geophys. Res., 102, 27 207-27 216, 1997.

Bailey, G. J., R. Sellek, and Y. Rippeth, A modeling study of the equatorial topside ionosphere, Ann. Geophysicae., 11, 263-272, 1993.

Balan, N., Y. Otsuka, G. J. Bailey, and S. Fukao, Equinoctial asymmetries in the ionosphere and thermosphere observed by the MU radar, J. Geophys. Res., 103, 9481-9495, 1998.

Essex, E. A., Equinoctial variations in the total electron content of the ionosphere at Northern and Southern Hemisphere stations, J. Atmos. Terr. Phys., 39, 645-650, 1977.

Feichter, E., and R. Leitinger, A 22-year cycle in the F layer ionization of the ionosphere, Ann. Geophysicae, 15, 1015-1027, 1997.

Fuller-Rowell, T. J., “The Thermospheric spoon”: a mechanism for the semiannual density variation, J. Geophy. Res., 103, 39513956, 1998.

Fuller-Rowell, T. J., and D. Rees, A three dimensional time dependent global model of the thermosphere, J. Atmos. Sci., 37, 2545, 1980.

Hirao, K., and K. I. Oyama, An improved type of electron temperature probe, J. Geomagn. Geoelectr., 21, 393-402, 1970.

Millward, G. H., R. J. Moffett, S. Quegan, and T. J. Fuller-Rowell, A coupled thermosphere-ionosphere-plasmasphere model (CTIP), in STEP Handbook on Ionospheric Models, Ed. R. W. Schunk, Utah State University, 239-279, 1996a.

Millward, G. H., H. Rishbeth, T. J. Fuller-Rowell, A. D., Aylward, S. Quegan, and R. J. Moffett, Ionospheric F2 layer seasonal 
and semiannual variations, J. Geophys. Res., 101, 5149-5156, 1996b.

Quegan S., G. J. Bailey, R. J. Moffett, R. A. Heelis, T. J. FullerRowell, D. Rees, and R. W. Spiro, A theoretical study of the distribution of ionization in the high-latitude ionosphere and the plasmasphere: first results on the mid-latitude trough and the light ion trough, J. Atmos. Terr. Phys., 44, 619, 1982.

Su, Y. Z., K.-I. Oyama, G. J. Bailey, T. Takahashi, and S. Watanabe, Comparison of satellite electron density and temperature measurements at low latitudes with a plasmasphereionosphere model, J. Geophy. Res., 100, 14 591-14 604, 1995.

Su, Y. Z., G. J. Bailey, K.-I. Oyama, and N. Balan, A modelling study of the longitudinal variations in the north-south asymmetries of the ionospheric equatorial anomaly, J. Atmos. Terr. Phys., 59, 1299-1310, 1997.
Su, Y. Z., G. J. Bailey, and K.-I. Oyama, Annual and seasonal variations in the low-latitude topside ionosphere, Ann. Geophysicae, 16, 974-985, 1998.

Titheridge, J. E., and M. J. Buonsanto, Annual variations in the electron content and height of the F layer in the Northern and Southern Hemispheres, related to neutral composition, J. Atmos. Terr. Phys., 45, 683-696, 1983.

Torr, M. R., and D. G. Torr, The seasonal behaviour of the F2layer of the ionosphere, J. Atmos. Terr. Phys., 35, 2237-2251, 1973.

Watanabe, S., and H. Oya, Occurrence characteristics of low latitude ionosphere irregularities observed by impedance probe on board the Hinotori satellite, J. Geomagn. Geoelectr., 38, 125 149, 1986. 